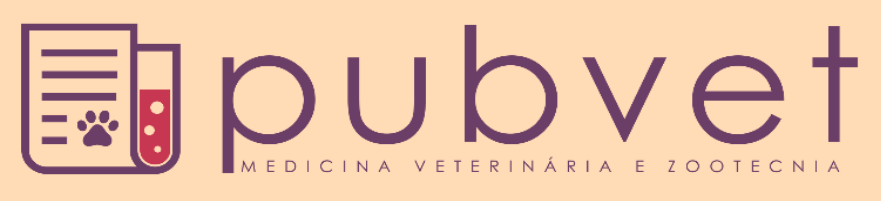

https://doi.org/10.31533/pubvet.v15n05a820.1-12

\title{
Prevalência e perfil de cães positivos para leishmaniose em um hospital veterinário do Rio Grande do Sul (2017-2019)
}

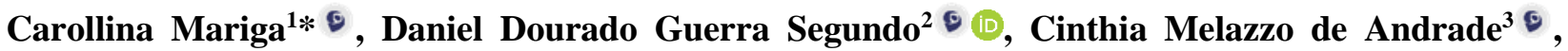 \\ Alexandre Krause $^{30}$, Saulo Tadeu Lemos Pinto Filho ${ }^{30}$ \\ ${ }^{I}$ Graduanda de Medicina Veterinária na Universidade Federal de Santa Maria. Santa Maria - RS Brasil. \\ ${ }^{2}$ Mestrando do Programa de Pós-Graduação e Pesquisa em Medicina Veterinária, Departamento de Clínica Médica e Cirurgia \\ de Pequenos Animais. Santa Maria - RS Brasil. \\ ${ }^{3}$ Professor da Universidade Federal de Santa Maria, Departamento de Clínica e Cirurgia de Pequenos Animais. Santa Maria - RS Brasil. \\ *Autor para correspondência, E-mail: carollina.mariga@hotmail.com
}

Resumo. Este estudo teve como objetivo classificar o perfil clínico dos cães acometidos por leishmaniose no Hospital Veterinário Universitário da Universidade Federal de Santa Maria (HVU-UFSM). Para isso, foram revisados históricos clínicos dos animais atendidos no HVUUFSM no período de janeiro de 2017 a julho de 2019, por meio dos prontuários dos pacientes. Neste período, 250 animais foram suspeitos para a doença, com resultado positivo para 61 $(24,4 \%)$ animais. O perfil clínico foi classificado conforme raça, gênero, idade, estado de vacinação e, então, separados de acordo com os sistemas envolvidos: digestório, neuromuscular, multissistêmico, cardiovascular, respiratório, linfático, oftálmico, urinário e tegumentar; sinais clínicos e a presença de doenças concomitantes. Este estudo revelou que a leishmaniose acomete mais animais sem raça definida (SRD), machos com dois a cinco anos de idade e acomete principalmente o sistema tegumentar, seguido do multissistêmico.

Palavras-chave: Canino, leishmaniose, perfil clínico, sinais clínicos, sistemas

\section{Prevalence and profile of positive dogs for leishmaniasis in a veterinary hospital of Rio Grande do Sul (2017-2019)}

\begin{abstract}
This study aimed to classify the clinical profile of dogs affected by leishmaniasis at the Veterinary Hospital of Universidade Federal de Santa Maria (HVU-UFSM). The clinical histories of the animals seen at the hospital from January 2017 to July 2019 were reviewed, using the patients' medical records. During this period, 250 animals were suspected to have leishmaniasis and $61(24,4 \%)$ of the animals tested positive. The clinical profiles were classified according to breed, gender, age, and vaccination status, then further separated according to the clinical signs the presence of concomitant diseases, and bodily systems involved: digestive, neuromuscular, multisystemic, hematopoietic, respiratory, lymphatic, ophthalmic, urinary and cutaneous. This study revealed that leishmaniasis most affects animals of mixed breed, particularly two to five years old males. The disease most often occurs in the cutaneous system, followed by multisystem presentation.
\end{abstract}

Keywords: Canine, clinical profile, clinical signs, leishmaniosis, systems 


\section{Introdução}

A leishmaniose é considerada pela Organização Mundial da Saúde como uma das seis doenças infecciosas mais importantes (FNS, 2010). É uma doença endêmica em 88 países tropicais, sendo que apenas seis países possuem 90\% dos casos mundiais da doença, sendo o Brasil um deles (Gontijo \& Melo, 2004; Marcondes \& Rossi, 2013; Ribeiro et al., 2019). Segundo Barbosa et al. (2010), estudos epidemiológicos revelam um aumento de casos de leishmaniose canina seguidos de leishmaniose humana. Segundo Oliveira \& Fernandes (2014), para cada um caso de leishmaniose humana, estima-se uma média de 200 cães infectados.

Souza et al. (2009) afirmam que o primeiro relato de leishmaniose autóctone aconteceu no ano de 2002 em Porto Alegre; enquanto, a Secretaria da Saúde do Rio Grande do Sul (2011) afirmou que apenas em 2008 houve diagnóstico de leishmaniose canina e o primeiro relato da presença do vetor no estado. Enquanto o diagnóstico de leishmaniose humana foi realizado apenas em 2009. Entretanto, segundo Pocai et al. (1998), o primeiro diagnóstico de leishmaniose visceral canina em Santa Maria foi realizado em 1985, com mais relatos da região. Nesta mesma época, estudos epidemiológicos realizados na região, não revelaram a presença do protozoário nem do flebótomo na época.

A leishmaniose é uma enfermidade infecciosa protozoótica de caráter crônico, causada pelo gênero Leishmania (Lonardoni et al., 2006; Oliveira \& Fernandes, 2014). A doença é transmitida pelas espécies de flebotomíneos hematófagos. A importância dos cães nos ciclos silvestre e urbano como principal hospedeiro é devido a alta taxa de reprodução da espécie, abandono e facilidade de adaptação e disseminação do vetor no meio, elevando as chances de uma epidemia (Silva \& Winck, 2018). Além do mais, o fechamento do diagnóstico a partir dos sinais clínicos e suas combinações é difícil já que é comum em outras enfermidades da rotina veterinária (Secretaria da Saúde, 2011). Este estudo teve como objetivo classificar o perfil clínico dos cães acometidos por leishmaniose no Hospital Veterinário Universitário de Santa Maria/RS, Brasil.

\section{Material e métodos}

Foram revisados os históricos clínicos dos animais atendidos no Hospital Veterinário Universitário (HVU) de uma instituição de Ensino Superior do Rio Grande do Sul no período de janeiro de 2017 a julho de 2019. Os animais suspeitos de leishmaniose foram testados para detecção de anticorpos, através teste imunocromatográficos rápidos $\left(I D E X X^{\circledR}\right.$ e Alere $\left.^{\circledR}\right)$. O teste da IDEXX ${ }^{\circledR}$ é pelo teste de ELISA, que utiliza conjugado e substrato exclusivos que amplificam os resultados e fornecem o diagnóstico em apenas seis minutos, enquanto que o teste da Alere ${ }^{\circledR}$ detecta anticorpos anti-Leishmania infantum, fornecendo o diagnóstico em 20 minutos. Os pacientes positivos foram classificados conforme raça, gênero, idade, estado de vacinação e, então, separados de acordo com os sinais clínicos dos sistemas orgânicos envolvidos (digestório, neuromuscular, multissistêmico, cardiovascular, respiratório, linfático, oftálmico, urinário, tegumentar), e doenças concomitantes. Os animais foram divididos de acordo com a faixa etária, sendo: adultos jovens, de um a quatro anos de idade; adultos velhos, de cinco a nove anos de idade e idosos, animais acima de 9 anos de idade.

As análises estatísticas foram realizadas utilizando o software Action Stat Pro (Estatcamp, São Carlos, Brasil). O teste de Qui-Quadrado de Yates foi empregado para avaliar a influência dos fatores idade e gênero dos cães positivos pelo teste rápido imunocromatográfico, sobre a proporção da ocorrência de sinais clínicos envolvendo os sistemas: digestório, neuromuscular, multissistêmico, respiratório, linfático, oftálmico, urinário e tegumentar. Para todas as análises, foi utilizado o nível de significância de $\mathrm{P}<0,05$.

\section{Resultados}

Foram atendidos no HVU-UFSM, no período de janeiro de 2017 a julho de 2019, um total de 11.910 animais. Destes, 250 (2,1\%) foram suspeitos de leishmaniose, e destes, 24,4\% (61/250) foram positivos no SNAP test. Os materiais usados para os exames foram: 89,9\% sangue total e 10,1\% soro. Quanto as raças mais acometidas, 57,4\% (35/61) eram SRD, 42,6\% (26/61) eram das seguintes raças definidas: seis Labrador 
Retriever, três Dachshund, dois Pug, Rottweiller, Akita Inu e um representante para Yorskshire Terrier, Dogue alemão, Dogo Argentino, Border Collie, Boiadeiro-australiano, Pastor-alemão, Cimarron, American pitt bull Terrier, Pinscher, Buldogue francês e Boxer. Em relação ao sexo, 44,3\% (27/61) eram fêmeas e $55,7 \%(34 / 61)$ machos.

A distribuição de acordo com a faixa etária foi de 49,2\% (20/61) de adultos jovens; 36,1 (22/61) de adultos velhos e 9,8\% (6/61) de idosos (Figura 1). Três animais não possuíam este dado na ficha cadastral. Não houve relatos da doença em animais filhotes com menos de um ano de idade. Com uma distribuição etária de seis animais com 1 ano de idade, dez com 2 anos, cinco com 3 anos, nove com 4 anos, sete com 5 anos, quatro com 6 anos, dois com 7 anos, seis com 8 anos, três com 9 anos, um com 10 anos, um com 11 anos, dois com doze anos e um com 14 anos de idade.

O estado sanitário revelou que $44(72,1 \%)$ animais não estavam com a vacinação em dia, enquanto apenas $12(19,7 \%)$ dos animais apresentavam programa de vacinação atualizada.

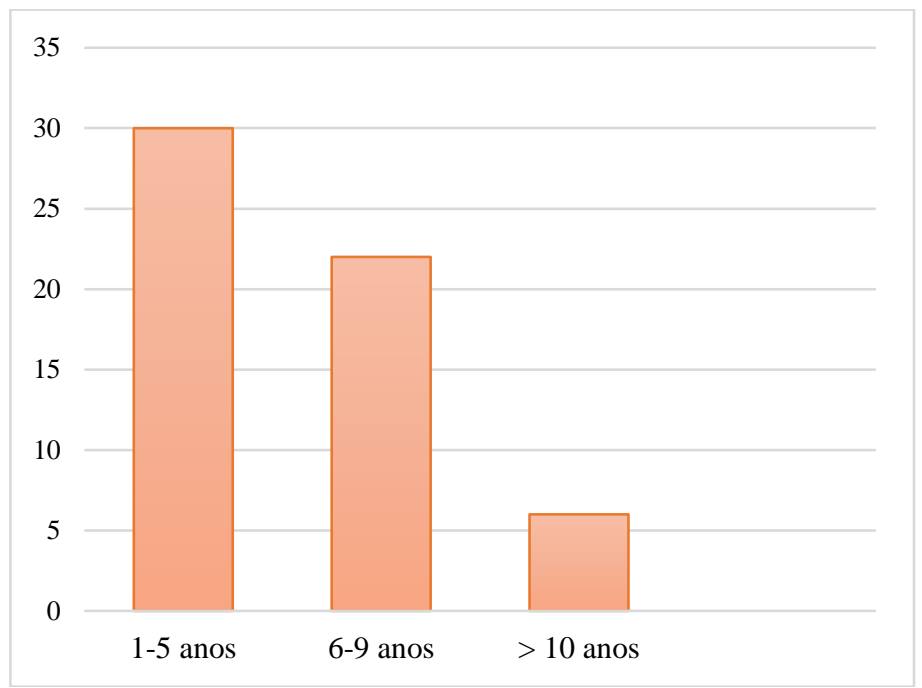

Figura 1. Distribuição etária dos animais positivos para leishmaniose no HVUUFSM de janeiro 2017 a julho de 2019 em números absolutos.

O sistema tegumentar foi o sistema mais acometido, presente em 64,3\% (36/56) dos casos clínicos. Os sinais clínicos de maior prevalência foram lesões de pele com 28,6\% (16/56) (Figura 2), sendo que a orelha foi o local mais acometido, em $50 \%$ dos animais, lesões generalizadas e na região periocular tiveram prevalência de $18,7 \%$ e apenas um dos animais apresentou lesões no focinho $(6,2 \%)$. O segundo sinal clínico mais presente foi a hipotricose, presente em 17,9\% (10/56), sendo visível nas áreas da cauda, perilabial, axila, orelha, nariz e periorbital.

A alopecia foi registrada na região perilabial e periocular dos dois animais afetados. O segundo sistema mais acometido foi o multissistêmico, com prevalência em 57,1\% (32/56) dos casos clínicos. Os sinais clínicos de maior presença foram emagrecimento progressivo com 47,2\% (27/56) e apatia com 16,1\% $(9,56)$. Os demais sinais clínicos estão listados na Figura 3.

Os sinais digestórios ocorreram em 39,3\% (22/56) dos pacientes, sendo o sinal clínico de maior prevalência a anorexia e o vômito com 14,3\% (8/56). Logo após vieram os sistemas linfático 37,5\% (21/56) com linfadenomegalia, presente em todos os casos, e oftálmico 28,6\% (16/56), com secreção ocular em $23,2 \%$ (13/56). Os demais sinais clínicos dos sistemas anteriores e os demais acometidos e respectivos sinais clínicos estão listados na tabela 1. A prevalência do sistema neuromuscular foi de 26,8\% (15/56), respiratório com 8,9\% (5/56), cardiovascular com 5,4\% (3/56), urinário com 5,4\% (3/56) e genital com $3,6 \%(2 / 56)$ dos casos. 


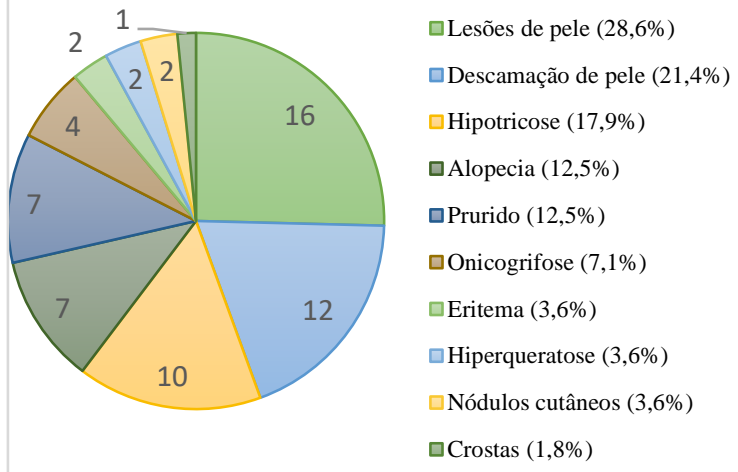

Figura 2. Prevalência dos sinais clínicos do sistema tegumentar dos animais positivos para leishmaniose no HVU-UFSM de janeiro 2017 a julho de 2019

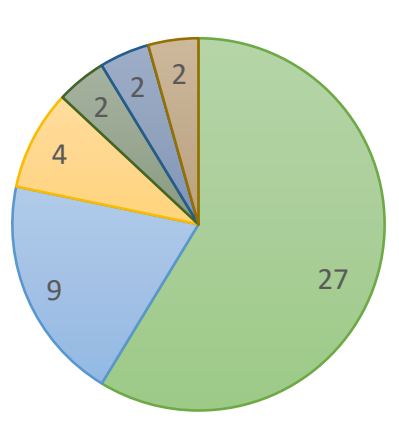

$\square$ Emagrecimento progressivo $(47,2 \%)$

$\square$ Apatia $(16,1 \%)$

$\square$ Hipertermia $(7,1 \%)$

口Caquexia $(3,6 \%)$

口Hipotermia $(3,6 \%)$

口Prostração $(3,6 \%)$

Figura 3. Prevalência dos sinais clínicos do multissistêmico dos animais positivos para leishmaniose no HVUUFSM de janeiro 2017 a julho de 2019.

Tabela 1. Prevalência dos demais sistemas e sinais clínicos relacionados aos animais positivos para leishmaniose no HVU-UFSM de janeiro de 2017 a julho de 2019.

\begin{tabular}{|c|c|c|c|}
\hline Sistema acometido & Sinal clínico & Número absoluto, x/56 & Número relativo, $\%$ \\
\hline \multirow[t]{10}{*}{ Digestório } & Anorexia & 8 & 14,3 \\
\hline & Vômito & 8 & 14,3 \\
\hline & Hiporexia & 7 & 12,5 \\
\hline & Diarreia & 5 & 8,9 \\
\hline & Desidratação & 4 & 7,1 \\
\hline & Melena & 4 & 7,1 \\
\hline & Polidipsia & 3 & 5,4 \\
\hline & Adipsia & 1 & 1,8 \\
\hline & Hematoquezia & 1 & 1,8 \\
\hline & Úlcera oral & 1 & 1,8 \\
\hline Linfático & Linfadenomegalia & 21 & 37,5 \\
\hline \multirow[t]{3}{*}{ Oftálmico } & Secreção ocular & 13 & 23.2 \\
\hline & Lesão ocular & 6 & 10,7 \\
\hline & Cegueira & 1 & 1,8 \\
\hline \multirow[t]{10}{*}{ Neuromuscular } & Ataxia & 5 & 8,9 \\
\hline & Fraqueza & 4 & 7,1 \\
\hline & Abdome abaulado e enrijecido & 2 & 3,6 \\
\hline & Claudicação & 2 & 3,6 \\
\hline & Edema de membros & 2 & 3,6 \\
\hline & Aumento de volume cervical & 1 & 1,8 \\
\hline & Dor nas articulações & 1 & 1,8 \\
\hline & Fratura de membro & 1 & 1,8 \\
\hline & Síncope & 1 & 1,8 \\
\hline & Tumor de membro & 1 & 1,8 \\
\hline \multirow[t]{4}{*}{ Respiratório } & Dispneia & 2 & 3,6 \\
\hline & Tosse & 2 & 3,6 \\
\hline & Epistaxe & 1 & 1,8 \\
\hline & Espirros & 1 & 1,8 \\
\hline \multirow[t]{2}{*}{ Cardiovascular } & Mucosas pálidas & 3 & 5,3 \\
\hline & Sopro anêmico & 1 & 1,8 \\
\hline \multirow[t]{4}{*}{ Urinário } & Débito urinário baixo & 1 & 1,8 \\
\hline & Incontinência urinária & 1 & 1,8 \\
\hline & Poliúria & 1 & 1,8 \\
\hline & Uremia & 1 & 1,8 \\
\hline \multirow[t]{2}{*}{ Genital } & Sangramento peniano & 1 & 1,8 \\
\hline & Sangramento vulvar & 1 & 1,8 \\
\hline
\end{tabular}

Os linfonodos acometidos nos animais com linfadenomegalia foram 71,4\% (15/21) do poplíteo, 47,6\% (10/21) do mandibular, 14,3\% (3/21) para inguinal, submandibular e pré-escapular. Um dos animais apresentava hemoptise. Quatorze $(22,9 \%)$ animais foram eutanasiados no HVU-UFSM e quatro $(6,6 \%)$ 
vieram à óbito. Quatro animais foram positivos para leishmaniose por haver contato com outros animais também positivos. As doenças concomitantes à leishmaniose foram: quatro animais com doença renal, sendo dois já em estado crônico, um com conjuntivite, um com cistite, um com dermatopatia, sem especificação, um com ectoparasita, sem especificação, e um com cardiopatia, também sem especificação da afecção acometida.

Apenas $42(75,0 \%)$ dos 56 animais positivos sintomáticos realizaram exames hematológicos. No eritrograma, 64,3\% (27/42) apresentaram anemia, sendo que dois animais receberam transfusão sanguínea e dois apresentavam mucosa pálida no exame físico. Ainda no hemograma, houve prevalência de $26,2 \%$ (11/42) de alta concentração de proteínas totais enquanto 7,1\% (3/42) apresentaram baixa concentração. No leucograma, 9,5\% (4/42) apresentaram leucocitose, enquanto apenas um paciente $(2,4 \%)$ apresentou leucopenia. A trombocitopenia esteve presente em 30,9\% (13/42) dos casos, favorecendo a presença de sangramentos, como os relatados neste artigo: hemoptise, melena, Hematoquezia, epistaxe, sangramento peniano e sangramento vulvar. O bioquímico apresentou baixos níveis de albumina em 61,9\% (26/42) dos animais, creatinina alta em 35,7\% (15/42), fosfatase alcalina alta em 35,7\% (15/42), ureia alta em 30,9\% (13/42), ALT (Alanina aminotransferase) baixa em 19,0\% (8/42), ureia baixa em 9,5\% (4/42), fósforo alto em 4,8\% (2/42), glicose alta em 2,4\% (1/42), amilase alta em 2,4\% (1/42) e ALT alta em 2,4\% (1/42). A urinálise apresentou proteinúria em 9,5\% (4/42) casos. A referência dos parâmetros hematológicos e bioquímicos utilizados no HVU-UFSM é de acordo com Kaneco e Silva (1997).

As análises estatísticas realizadas para avaliar a influência dos fatores de gênero e idade dos cães positivos pelo teste rápido imunocromatográfico, sobre a proporção da ocorrência de sinais clínicos envolvendo os sistemas: digestório, neuromuscular, multissistêmico, respiratório, linfático, oftálmico, urinário e tegumentar estão listadas na tabela 2. Nenhum resultado apresentou significância estatística. Para todas as análises, foi utilizado o nível de significância de $\mathrm{P}<0,05$.

Tabela 2. Resultados do teste de Qui-Quadrado de Yates para avaliar a influência dos fatores idade e gênero sobre a proporção da ocorrência de sinais clínicos envolvendo os sistemas orgânicos

\begin{tabular}{lcc}
\hline Sistema acometido & Gênero & Idade \\
\hline Digestório & $\mathrm{X}^{2}=0,441$ & $\mathrm{X}^{2}=4,213$ \\
Hematopoiético & $\mathrm{X}^{2}=0,631$ & $\mathrm{X}^{2}=0,589$ \\
Linfático & $\mathrm{X}^{2}=1,353$ & $\mathrm{X}^{2}=3,538$ \\
Multissistêmico & $\mathrm{X}^{2}=0,737$ & $\mathrm{X}^{2}=3,998$ \\
Neuromuscular & $\mathrm{X}^{2}=4,962$ & $\mathrm{X}^{2}=8,119$ \\
Ocular & $\mathrm{X}^{2}=0,465$ & $\mathrm{X}^{2}=2,459$ \\
Respiratório & $\mathrm{X}^{2}=0,534$ & $\mathrm{X}^{2}=2,437$ \\
Tegumentar & $\mathrm{X}^{2}=2,582$ & $\mathrm{X}^{2}=3,944$ \\
Urinário & $\mathrm{X}^{2}=6,568$ & $\mathrm{X}^{2}=2,144$ \\
\hline
\end{tabular}

\section{Discussão}

A leishmaniose é uma doença infecciosa parasitária de caráter crônico, causada pelo protozoário do gênero Leishmania (Carvalho et al., 2002). A transmissão se dá por meio da picadura de fêmeas de insetos flebotomíneos infectados, prioritariamente por Lutzomyia longipalpis (Santos et al., 1998). Possui importância em saúde pública, pois o homem pode entrar no ciclo silvestre ou urbano, tornando-se uma antoprozoonose (Secretaria da Saúde, 2011).

Segundo Monteiro (2011), até os anos 2000 apenas o Rio Grande do Sul não era considerado uma área de risco de transmissão de Leishmaniose, até que em 2002 foi relatado o primeiro caso autóctone humano em Porto Alegre. Entretanto, a Secretaria da Saúde (2011) afirmou que o RS era livre até 2008, quando houve casos autóctones em cães (2008) e humano (2009) e o primeiro relato da presença do vetor (2008) no estado, tornando-se área de transmissão desta enfermidade. Segundo o Ministério da Saúde (2019), apenas 0,2\% dos casos de Leishmaniose visceral humana, entre os anos de 2003-2009, eram registrados na região sul. 
De acordo com a Secretaria da Saúde do RS (2011), Porto Alegre possuía uma soroprevalência de 4,1\% em 2010. Nos anos de 2009-2010, cerca de 20,8\% dos cães eram soro reagentes em 34 municípios do Rio Grande do Sul, próximo à prevalência relatada neste artigo (24,4\%). Entretanto, Bustamante (2008) revelou diferentes prevalências de acordo com o teste utilizado, sendo que, em cães, a prevalência no estado do RS é de $5,1 \%$ no teste ELISA e $23 \%$ no teste de imunofluorescência indireta (IFI). O SNAP test da IDEXX ${ }^{\circledR}$ utilizado nesse estudo possui 99,2\% de especificidade e 96,3\% de sensibilidade, enquanto o da Alere ${ }^{\circledR}$ possui 99,8\% e 97,2\% de especificidade e sensibilidade respectivamente, embora, ambos podem resultar em falsos negativos. Segundo Pocai et al. (1998), o primeiro diagnóstico de leishmaniose visceral canina em Santa Maria foi realizado em 1985, com mais relatos da região. Nesta época, estudos epidemiológicos realizados na região de Itaara, cidade vizinha à Santa Maria, não revelaram a presença do protozoário nem do flebótomo na época. Em 2003, um levantamento em cães apresentou todas as 203 amostras negativas através o teste de IFI para Leishmaniose canina em Santa Maria e região (Marcondes \& Rossi, 2013).

Segundo um estudo realizado por França-Silva et al. (2003) com 96,1\% da população canina de uma área endêmica em Minas Gerais revelou prevalência nas raças Boxer (24,6\%) e Cocker spaniel $(26,9 \%)$, enquanto 7,8\% dos animais sem raça definida (SRD) eram positivos. Mesmo tendo apenas um relato em um cão Boxer, a prevalência desta pesquisa é em animais SRD $(57,4 \%)$, provavelmente devido à população de cães atendidos no HVU-UFSM serem majoritariamente mestiços. Belo et al. (2013) ainda afirmam que as variáveis com correlação significante foram animais de pelo curto, raça pura, cães peridomésticos e presença de áreas verdes próximas à residência.

Segundo França-Silva et al. (2003), a razão entre macho e fêmea era de 1,6:1,0 na área que é considerada endêmica em Minas Gerais, correlação semelhante aos valores de Santa Maria que apresenta 1,3:1 entre machos e fêmeas. Enquanto, Campos et al. (2017) e Figueiredo et al. (2014) também revelam uma maior prevalência em machos; porém, é comprovado estatisticamente que essa relação não demonstra uma significância estatística da prevalência entre os gêneros. Belo et al. (2013) afirmam uma prevalência maior em cães entre um a dois anos de idade, sem associação estatística significante. Enquanto, Figueiredo et al. (2014) afirmam que cães com menos de dois anos de idade possuem 7x mais chance de serem positivos com significância estatística. No presente estudo, a maior prevalência se deu em animais adultos jovens, entre um a quatro anos de idade, com uma distribuição etária de $16,4 \%$ dos animais com dois anos de idade; $14,7 \%, 4$ anos de idade e 11,5\% com cinco anos de idade. Segundo Medeiros et al. (2009), a maior incidência em cães jovens pode ser justificada pela imaturidade imunológica, enquanto que nos animais adultos ocorrem dois picos de incidência da doença, um antes dos três anos e outra entre oito aos dez anos de idade.

Segundo o Ministério da Saúde (2019), a leishmaniose visceral é considerada uma doença em expansão no Brasil, sendo que até meados da década de 80 a leishmaniose era restrita ao nordeste e, atualmente, todas as regiões do país confirmam novos casos todos os anos. Segundo Amábilli (2018), no Brasil há a disponibilização de uma vacina contra leishmaniose canina, aprovada pelo MAPA e pelo Ministério da Saúde, que induz resposta imune celular a níveis elevados de IFN- $\alpha$ e humoral com produção de anticorpo específico do antígeno vacinal, sem reagir com as fases de promastigotas da infecção natural, sendo possível diferenciar animais vacinados dos animais infectados naturalmente. Segundo Ministério da Saúde, o uso da vacina é apenas para proteção individual de cães assintomáticos e com resultados negativos para exames sorológicos, enquanto cães sintomáticos e/ou positivos são passíveis às medidas sanitárias vigentes. Neste estudo, apenas $19,7 \%$ dos cães positivos possuíam programa de vacinação em dia, sendo informações sobre a vacina polivalente, não específica para leishmaniose, somado a isso, Tavares et al.( 2009) relatam que $45 \%$ da sua população de estudo era vacinada apenas para raiva.

De acordo com a lei nacional N. ${ }^{\circ} 1.738$-A, de 2011, art. $4^{\circ}$ “cães e gatos infectados pela leishmaniose poderão receber tratamento em clínicas particulares". Mesmo que a OMS, Organização Mundial da Saúde, recomende a eutanásia de cães soropositivos como medida de controle, a entidade ainda reconhece a dificuldade desta extrema decisão, afirmando que podem necessárias medidas alternativas para suprir as necessidades de controle, diminuindo o ônus emocional sobre a sentença de morte ao membro de uma família. Lopes et al. (2019) e Simão (2018) afirmam que a educação, conhecimento e conscientização da 
população pela iniciativa das autoridades sanitárias e profissionais da saúde visando prevenção da disseminação da doença é a melhor forma de prevenção, além de extinguir métodos primitivos e desumanos de combate à doença, como a eutanásia em massa de cães soropositivos. Segundo Hnilica \& Patterson (2017), os sinais clínicos de gatos acometidos pela leishmaniose são nódulos locais com possível ulceração, principalmente no pavilhão auricular, pálpebras, lábios ou focinho, enquanto que as infecções disseminadas são raras.

Segundo o Ministério da Saúde (2019), um estudo de avaliação da efetividade de coleiras com deltametrina a $4 \%$ revela diminuição da prevalência de leishmaniose visceral nas áreas utilizadas. Entretanto, Alves et al. (2018) revelam dificuldades operacionais no uso de coleiras caninas, principalmente pela alta taxa de perda de coleiras e domicílios fechados durante as visitas, além de animais não encontrados, sumidos, doados e vendidos. Contudo, o mesmo estudo anterior revelou que após o primeiro ciclo da pesquisa, dos animais possíveis de reavaliação, 78,6\% foram soronegativos. Dos animais infectados no primeiro ciclo, 76,6\% foram eutanasiados. O uso de inseticidas residuais é considerado eficiente para reduzir população de flebótomos e, consequente, redução da transmissão parasitária; porém, é um método que exige programa contínuo.

Segundo a Secretaria da Saúde do RS (2019), as manifestações clínicas são variáveis e dependentes da resposta imunológica do animal infectado. Segundo Sociedade Mundial de Proteção Animal (2015), cerca de $10 \%$ dos cães possuem resistência natural à infecção, logo, não desenvolvem a doença devido à forte resposta imunológica. Segundo Silva (2007), a leishmaniose visceral tem evolução lenta e início insidioso, variando de animais aparentemente normais à quadros graves, apresentando debilidade e caquexia. Enquanto a forma cutânea é caracterizada pelas áreas alopecias, úlceras, hiperqueratose, descamação furfurácea, áreas inflamatórias e hiperqueratose, principalmente no focinho, ao redor dos olhos, orelhas e extremidades (Sociedade Mundial de Proteção Animal, 2015).

Conforme os sinais clínicos apresentados, os animais são classificados em: 1. Assintomáticos, os cães que não apresentam manifestações clínicas, mas são soropositivos, portanto, são de importância em saúde pública por serem fontes de infecção de difícil identificação; 2. Oligossintomáticos, cães que apresentam discreta perda de peso, pelo opaco e linfadenomegalia, principalmente do poplíteo ou submandibulares, levantando a suspeita da doença; 3. Sintomáticos, cães que manifestam todos ou os sinais clínicos mais característicos da doença, como alopecia, eczema furfuráceo, úlceras, hiperqueratose, onicogrifose, emagrecimento, caquexia, ceratoconjuntivite, linfadenomegalia, paresia dos membros posteriores e hepatoesplenomegalia (Silva, 2007). Segundo a Sociedade Mundial de Proteção Animal (2015), mais de $50 \%$ dos animais infectados permanecem assintomáticos durante os dois primeiros anos da infecção, enquanto Silva (2007) afirma que a forma assintomática é cerca de 40 a $60 \%$ dos animais infectados no Brasil. Nesta pesquisa, dos 61 animais positivos no SNAP test, 56 animais eram sintomáticos, sendo apenas $5(8,2 \%)$ assintomáticos.

Segundo Torres Neto et al.(2008), a leishmaniose possui 4 fases distintas, sendo elas: 1. Período discreto, com linfadenopatia discreta ou dermatite papular; 2. Período moderado, com dermatite esfoliativa difusa, ulcerações, anorexia, perda de peso e epistaxe; 3. Período grave, com deposição de imunocomplexos, resultando em vasculites, artrites, uveíte e glomerulonefrite; 4. Período muito grave, com tromboembolismo pulmonar, síndrome nefrótica e estádio final de doença renal crônica. As diferentes espécies de Leishmania caracteriza formas variadas de apresentação clínica da doença (Abbiati et al., 2019).

Segundo Pocai et al. (1998), os sinais clínicos mais presentes nos cinco animais diagnosticados com leishmaniose visceral na região de Santa Maria foram: icterícia, anorexia, vômito, emaciação, febre intermitente, linfadenopatia, esplenomegalia e hematúria. Já na necropsia, apresentaram sinais de doença crônica como: icterícia, anorexia, vômito, febre intermitente e emaciação, alguns sinais foram registrados de acordo com o histórico clínico. No presente estudo houve relato com prevalência significativa de emagrecimento progressivo (47,2\%), linfadenomegalia $(37,5 \%)$, lesões de pele $(28,6 \%)$ e secreção ocular $(23,2 \%)$, enquanto que anorexia e vômito tiveram prevalência em $14,3 \%$ dos pacientes. 
De acordo com a Sociedade Mundial de Proteção Animal, alterações neurológicas também são relacionadas à leishmaniose canina, sendo paresia dos membros, ataxia e convulsão. $\mathrm{O}$ sistema neuromuscular foi o quinto sistema mais acometido nesta pesquisa, sendo que foi representado principalmente pela ataxia $(8,9 \%)$ e relatos de outros sinais também citados por outros autores como fraqueza, prostração e síncope (Lappin et al., 2015).

Figueiredo et al. (2014) relataram uma prevalência de $10 \%$ pela sorologia dos pacientes caninos com perda de peso, 14,4\% alterações cutâneas, $10 \%$ alterações oculares, 9,1\% linfadenomegalia e 23,3\% onicogrifose. No atual estudo, a taxa de emagrecimento foi de $47,2 \%$, sendo bem acima do autor acima, as alterações cutâneas e oculares foram diversas e possuíram prevalência de 64,3\% e 28,6\%, respectivamente. Entretanto, a taxa de onicogrifose foi apenas de 7,1\% nesta pesquisa. Segundo Lappin et al. (2015), sinais comumente identificados no exame físico e também verificados no presente estudo são diarreia $(8,9 \%)$, melena $(7,1 \%)$, polidipsia $(5,4 \%)$, hipertermia $(7,1 \%)$, tosse $(3,6 \%)$, epistaxe $(1,8 \%)$, espirros $(1,8 \%)$ e poliúria $(1,8 \%)$.

A forma cutânea é representada principalmente por alopecia, descamação com aspecto eczematoso e furfuráceo, inflamatório e não pruriginoso, com a possibilidade de evoluir para nódulos, erosões e crostas. Pode haver contaminação secundária ou a presença de ectoparasitas como dermatófitos e ácaros. Também pode ser relatada a presença de dermatite, hiperqueratose e úlceras na pele (Silva, 2007). No presente estudo, as manifestações do sistema tegumentar foi a de maior prevalência, sendo por lesão de pele $(28,6 \%)$ como principal sinal clínico, presente em áreas da orelha, região periocular e focinho (Figura 4), além de se apresentar de forma generalizada por todo o corpo. Houve também relato da presença de alopecia (12,5\%), descamação da pele $(21,4 \%)$, eritemas e nódulos cutâneos $(3,6 \%)$ e crostas $(1,8 \%)$. Também presença de ectoparasitas $(3,6 \%)$. Além disso, houve relato de prurido em 12,5\% dos casos.

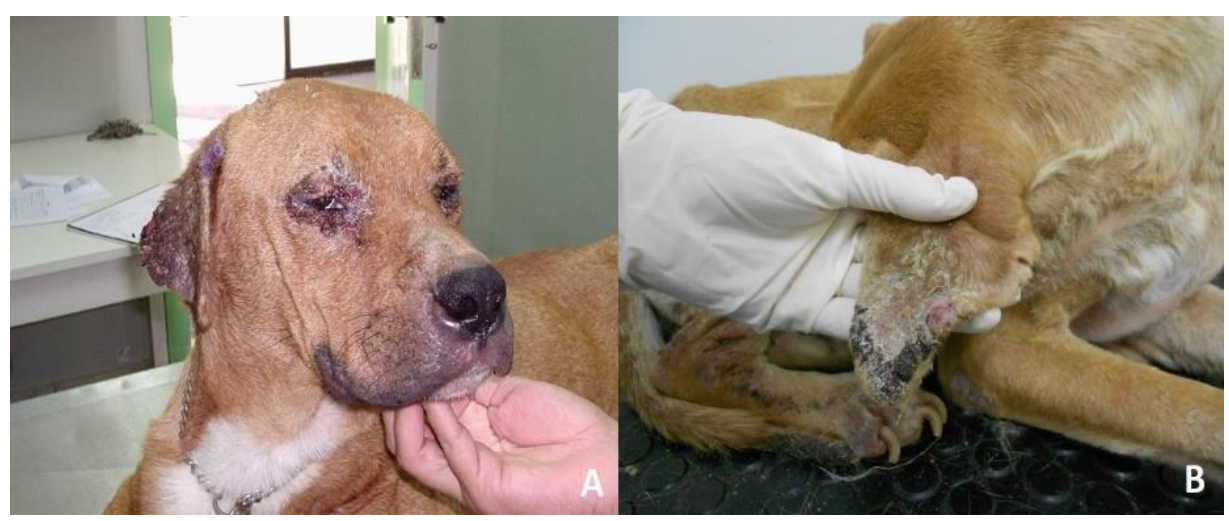

Figura 4. Leishmaniose clínica em cães. A. Alopecia e formação de crostas periocular e auricular, com aspecto opaco e quebradiço dos pelos do animal acometido por Leishmaniose; $\mathbf{B}$. Formação de crostas e área de descamação furfurácea na forma cutânea, detalhe do crescimento exagerado das unhas (onicogrifose). Fonte: Dra. Claudete Schmidt/UFSM.

Segundo a Sociedade Mundial de Proteção Animal (2015), as alterações oculares são principalmente ceratoconjuntivite, blefarite, uveíte, retinopatia e hifema. Sendo apenas o quinto sistema mais acometido, é compatível com WSPA apenas na presenta de ceratoconjuntivite em um cão acometido. De acordo com Figueiredo et al. (2014), cerca de $15 \%$ dos pacientes positivos possuíam linfadenopatia, todos acometidos no poplíteo, $35 \%$ alopecia, sendo que destes $71,42 \%$ periocular e $28,58 \%$ bilateral e todos os animais apresentavam descamação de pele. Já para Monteiro (2011), a linfadenomegalia acometeu principalmente poplíteo e inguinal. Em nosso estudo, o poplíteo foi acometido em 71,4\% dos casos de linfadenopatia, seguido do mandibular $(47,6 \%)$ e inguinal com $14,3 \%$. A alopecia teve baixa prevalência, presente apenas em $12,5 \%$ dos pacientes, nas regiões perilabial e periocular. Entretanto, a presença de hipotricose esteve em 17,9\%, visíveis na cauda, perilabial, axila, orelha, nariz e periorbital. A descamação de pele também foi relatada neste estudo, em $21,4 \%$ dos casos. 
A Sociedade Mundial de Proteção Animal ressalta que os sinais de leishmaniose são inespecíficos, mimetizando diversas doenças sistêmicas. A Secretaria da Saúde (2011) reforça que a leishmaniose é uma doença de difícil diagnóstico clínico, já que a apresentação dos diversos sinais clínicos e suas combinações são tão comuns em outras enfermidades caninas mais frequentes na rotina clínica, como dermatoses, infecções generalizadas entre outras. Considerando que 50\% dos casos apresentam anemia, podendo apresentar tanto leucocitose quanto leucopenia. Segundo Sociedade Mundial de Proteção Animal, anemia, trombocitopenia e/ou leucopenia, alterações no proteinograma e diáteses hemorrágicas também são sinais da leishmaniose. Medeiros et al. (2008) revelaram uma prevalência de 60\% de anemia nos animais, enquanto este estudo apresentou um valor semelhante, sendo 64,3\% de animais anêmicos. A hiperproteinemia também é confirmada por Medeiros et al. (2008), que explica que a elevação é consequência da ativação policlonal de linfócitos B e aumento da produção de anticorpos. No leucograma, apenas 9,5\% apresentaram leucocitose, prevalência que vai de encontro com os achados de Almeida (2017), em que a leucocitose era a segunda alteração mais presente, e menor prevalência comparada aos 13,1\% relatado por Medeiros et al. (2008). Em contrapartida, o relato de trombocitopenia do presente estudo foi apenas de $30,9 \%$ dos casos enquanto Medeiros et al. (2008) chegou a 53,1\% dos animais acometidos. A trombocitopenia é secundária aos imunocomplexos circulantes que se ligam às plaquetas e causam acelerada destruição pelos macrófagos do sistema mononuclear fagocitário (Leonel et al., 2008).

Segundo Secretaria da Saúde (2019), a fase final é caracterizada por caquexia, inanição e óbito. A lesão renal se dá por deposição de imunocomplexos, com aumento significativo de creatinina, ureia, presença de hematúria e proteinúria nos exames laboratoriais. Apenas 3,6\% dos animais desta pesquisa apresentaram caquexia, porém $22,9 \%$ dos animais atendidos foram eutanasiados no HVU-UFSM e 6,6\% vieram à óbito durante o atendimento. Resultados compatíveis com lesão renal foram de 35,7\% para níveis elevados de creatinina e 30,9\% para ureia. Além de alterações bioquímicas hepáticas secundárias à hepatomegalia causada pela Leishmaniose teve prevalência de 61,9\% de hipoalbuminemia e 35,7\% com níveis elevados de fosfatase alcalina. A proteinúria esteve presente em $9,5 \%$ dos casos.

De acordo com o boletim epidemiológico do Ministério da Saúde (2019), é registrado de 0,7-1,3 milhões de novos casos por ano de leishmaniose em todo o mundo, sendo considerada pela OMS como uma das seis doenças infecciosas mais importantes em todo o mundo, devido ao alto coeficiente de detecção e capacidade de deformidades, visto que em a letalidade chega a $90 \%$ dos casos não tratados. Conforme a Secretaria da Saúde (2011), o programa de vigilância de leishmaniose é baseado em vigilância epidemiológica de casos humanos, dos reservatórios caninos e entomológica. As dificuldades deste estudo se deram ao histórico incompleto e à não especificidade dos sinais clínicos observados, principalmente na área de dermatologia, além da desatualização da situação da leishmaniose em Santa Maria e no estado do Rio Grande do Sul conforme o período deste estudo. Que o conhecimento fornecido através deste estudo possa ser usado em estratégias de intervenções adaptadas ao município e região de Santa Maria, que o poder público possa assegurar medidas de controle efetivas contra o combate desta zoonose.

\section{Conclusão}

Os resultados deste estudo permitem concluir que a prevalência da leishmaniose em cães testados no HVU-UFSM é de $24,4 \%$. O perfil clínico apresenta predisposição para animais sem raça definida e adultos jovens, de um a quatro anos de idade, seguido pelos adultos velhos, de cinco a nove anos de idade. $\mathrm{O}$ sistema mais acometido foi o tegumentar, sendo o sinal clínico mais presente as lesões de pele, seguido por sinais multissistêmicos, como emagrecimento progressivo.

\section{Conflito de interesse}

Declaramos que dentro dos últimos cinco anos e para o futuro próximo que não possuímos nenhum conflito de interesse de nenhuma ordem.

\section{Referências}

Abbiati, T. C., Freitas, D. M., Alves, L. C., Freitas, B. G., Rezende, R. S., Barbosa, S. G., Jorge, A. L. T. 
A., Santos, S. M., \& Lopes, M. C. (2019). Leishmaniose visceral canina: Relato de caso. PUBVET, 13, 1-8. https://doi.org/10.31533/pubvet.v13n4a307.1-8.

Almeida, V. A. (2017). Alterações na medula óssea e distúrbios hematológicos na leishmaniose visceral canina. Instituto Gonçalo Moniz.

Alves, E. B., Figueiredo, F. B., Rocha, M. F., \& Werneck, G. L. (2018). Dificuldades operacionais no uso de coleiras caninas impregnadas com inseticida para o controle da leishmaniose visceral, Montes Claros, MG, 2012. Epidemiologia e Serviços de Saúde, 27, e2017469. https://doi.org/10.5123/S167949742018000400001.

Amábilli, S. R. (2018). Leishmaniose visceral canina no município de Florianópolis: distribuição temporal e espacial. Curitibanos, SC.

Barbosa, D. S., Rocha, A. L., Santana, A. A., Souza, C. S. F., Dias, R. A., Costa-Júnior, L. M., \& AbreuSilva, A. L. (2010). Soroprevalência e variáveis epidemiológicas associadas à leishmaniose visceral canina em área endêmica no município de São Luís, Maranhão, Brasil. Ciência Animal Brasileira, 11(3), 653-659.

Belo, V. S., Struchiner, C. J., Werneck, G. L., Barbosa, D. S., de Oliveira, R. B., Neto, R. G. T., \& Silva, E. S. (2013). A systematic review and meta-analysis of the factors associated with Leishmania infantum infection in dogs in Brazil. Veterinary Parasitology, 195(1-2), 1-13.

Bustamante, M. C. F. S. (2008). Caracterização epidemiológica de município sem autoctonia para leishmaniose tegumentar americana. Universidade Federal Rural do Rio de Janeiro.

Câmara de Deputados. Projeto de Lei N. $^{\circ}$ 1.738-A, de 2011. https://www.camara.leg.br/proposicoesWeb/prop mostrarintegra;jsessionid=AB85E839A09D5AF86E 764E92C753BC70.proposicoesWebExterno2?codteor $=1670316 \&$ filename $=$ Avulso+PL+1738/2011\#: :text=Art.,pela\%20eutan\%C3\%A1sia\%20de\%20seu\%20c\%C3\%A3o.

Campos, R., Santos, M., Tunon, G., Cunha, L., Magalhães, L., Moraes, J., Ramalho, D., Lima, S., Pacheco, J.A., Limpscomb, M., Jesus, A.R. \& Almeida, R.P. (2017). Epidemiological aspects and spatial distribution of human and canine visceral leishmaniasis in an endemic area in northeastern Brazil. Geospatial Health, 12(503).

Carvalho, F. A., Charest, H., Tavares, C. A., Matlashewski, G., Valente, E. P., Rabello, A., Gazzinelli, R. T., \& Fernandes, A. P. (2002). Diagnosis of American visceral leishmaniasis in humans and dogs using the recombinant Leishmania donovani A2 antigen. Diagnostic Microbiology and Infectious Disease, 43, 289-295.

Figueiredo, M. J. F. M., Souza, N. F., Figueiredo, H. F., Meneses, A. M. C., Silva Filho, E., \& Nascimento, G. G. (2014). Fatores de risco e classificação clínica associados à soropositividade para Leishmaniose Visceral Canina. Ciência Animal Brasileira, 15(1), 102-106.

FNS. (2010). - Fundação Nacional de Saúde (M. de controle da leishmaniose tegumentar americana (ed.)). Ministério da Saúde.

França-Silva, J. C., Costa, R. T., Siqueira, A. M., Machado-Coelho, G. L. L., Costa, C. A., Mayrink, W., Vieira, E. P., Costa, J. S., Genaro, O., \& Nascimento, E. (2003). Epidemiology of canine visceral leishmaniosis in the endemic area of Montes Claros Municipality, Minas Gerais State, Brazil. Veterinary Parasitology, 111(2-3), 161-173.

Gontijo, C. M. F., \& Melo, M. N. (2004). Leishmaniose visceral no Brasil: quadro atual, desfios e perspectivas. Revista Brasileira de Epidemiologia, 7(3), 338-349.

Hnilica, K. A., \& Patterson, A. P. (2017). Autoimmune and immune-mediated skin disorders. In K. A. Hnilica \& P. A.P. (Eds.), Small animal dermatology: a color atlas and therapeutic guide. Elsevier.

IDEXX Brasil. SNAP leishmania. https://www.idexx.com.br/pt-br/veterinary/snap-tests/snap-leishmania/

Kaneco, J. J., Harvey, J.W. \& Bruss, M. L. (1997). Clinical biochemistry of domestic animals. $5^{\circ}$ ed. California: Academic 
Lappin, M. R., Chandrashekar, R., Stillman, B., Liu, J., \& Mather, T. N. (2015). Evidence of Anaplasma phagocytophilum and Borrelia burgdorferi infection in cats after exposure to wild-caught adult Ixodes scapularis. Journal of Veterinary Diagnostic Investigation, 27(4), 522-525.

Leonel, R. A. B., Matsuno, R. M. J., Santos, W., Veronezi, A. H. M., Costa, D. R., \& Sacco, S. R. (2008). Trombocitopenia em animais domésticos. Revista CCentífica Eletônica de Medicina Veterinária, 11.

Lonardoni, M. V. C., Silveira, T. G. V., Alves, W. A., Maia-Elkhoury, A. N. S., Membrive, U. A., Membrive, N. A., Rodrigues, G., Reis, N., Zanzarini, P. D., \& Ishikawa, E. (2006). Leishmaniose tegumentar americana humana e canina no Município de Mariluz, Estado do Paraná, Brasil. Cadernos de Saúde Pública, 22, 2713-2716.

Lopes, G. S., Sousa, V. A., Martins, J. S. C., Sousa, E. S., \& Cajaiba, R. L. (2019). Nível de conhecimento e medidas de prevenção de moradores sobre a Leishmaniose Visceral em área endêmica no Maranhão, Brasil. Archives of Health Investigation, 8(6), 285-289.

Marcondes, M., \& Rossi, C. N. (2013). Leishmaniose visceral no Brasil. Brazilian Journal of Veterinary Research and Animal Science, 50(5), 341-352.

Medeiros, C. M. O., Melo, A. G. C., Lima, A. K. F., Silva, I. N. G., Oliveira, L. C., \& Silva, M. C. (2008). Perfil hematológico de cães com leishmaniose visceral nomunicípio de Fortaleza, Ceará. Ciência Animal, 18(1), 43-50.

Medeiros, R. J., Monteiro, F. O., Castelo da Silva, G., \& Nascimento Júnior, A. (2009). Casos de intoxicações exógenas em cães e gatos atendidos na Faculdade de Veterinária da Universidade Federal Fluminense durante o período de 2002 a 2008. Ciência Rural, 39(7), 2105-2110.

Ministério da Saúde. (2019). Leishmaniose Visceral: o que é, causas, sintomas, tratamento, diagnóstico e prevenção. https://saude.gov.br/saude-de-a-z/leishmaniose-visceral

Monteiro, S. G. (2011). Parasitologia na medicina veterinária (Vol. 1). Roca.

Oliveira, A. R., \& Fernandes, C. A. (2014). Focos e fatores associados ao aparecimento de leishmaniose tegumentar americana (lta) e leishmaniose visceral (lv) no Cariri Cearense. Revista Interfaces: Saúde, Humanas e Tecnologia, 2(5).

Pocai, E. A., Frozza, L., Headley, S. A., \& Graça, D. L. (1998). Leishmaniose visceral (calazar): cinco casos em cães de Santa Maria, Rio Grande do Sul, Brasil. Ciência Rural, 28(3), 501-505.

Ribeiro, C. R., Gonçalves, C. A., Cruz, L. M., \& Galera, P. D. (2019). Prevalência da leishmaniose visceral canina e coinfecções em região periurbana no Distrito Federal-Brasil. Ciência Animal Brasileira, 20, 1-10.

Santos, S. O., Arias, J., Rribeiro, A. A., Hoffmann, M. P., Freitas, R. A., \& Malacco, M. A. F. (1998). Incrimination of Lutzomyia cruzi as a vector of American visceral leishmaniasis. Medical and Veterinary Entomology, 12(3), 315-317.

Secretaria da Saúde, RS. Leishmaniose Visceral no Rio Grande do Sul. Boletim epidemiológico, v.13, n.1, 2011. https://portalarquivos2.saude.gov.br/images/pdf/2019/setembro/25/boletim-especial-21ago19-web.pdf.

Secretaria de Vigilância em Saúde, Ministério da Saúde (2019). Vigilância em Saúde no Brasil 2003-2019: da criação da secretaria de vigilância em saúde aos dias atuais. Boletim Epidemiológico, número especial.

Silva, C. M. H. S., \& Winck, C. A. (2018). Leishmaniose visceral canina: revisão de literatura. Revista Da Universidade Vale do Rio Verde, 16(1), 1-12.

Silva, F. S. (2007). Patologia e patogênese da leishmaniose visceral canina. Revista Tropical - Ciencias Agrarias e Biologicas, 1(1), 20-31.

Simão, J. S. C. (2018). Tratamento e prevenção da leishmaniose em cães domésticos (Canis familiaris): avaliação de diferentes cenários. Universidade de Lisboa.

Sociedade Mundial de Proteção Animal. (2015). Leishmaniose Visceral Canina: Um manual para o clínico veterinária. WSPA Brasil, Copacabana, Rio de Janeiro

Souza, M. B., Carvalho, R. W., Machado, R. N. M., \& Wermelinger, E. D. (2009). Flebotomíneos de áreas 
com notificações de casos autóctones de leishmaniose visceral canina e leishmaniose tegumentar americana em Angra dos Reis, Rio de Janeiro, Brasil. Revista Brasileira de Entomologia, 53(1), 147-150.

Tavares, N. M., Santos, D. M., Oliveira, C. I., \& Brodskyn, C. I. (2009). Estratégias de vacinação contra leishmaniose visceral e cutânea: lições dos modelos experimentais. Gazeta Médica Da Bahia, 79.

Torres Neto, R., Rodrigues, M. M. P., Amorim, R. L., Conceição, L. G., Luvizotto, M. C. R., \& Franco, S. R. V. S. (2008). Padrões histopatológicos das lesões descamativas e ulcerativas da pele em cães com leishmaniose. Semina: Ciências Agrárias, 29(3), 667-676.

Histórico do artigo:

Recebido: 30 de novembro de 2020.

Aprovado: 14 de janeiro de 2021.

Disponível online: 20 de abril de 2021 .
Licenciamento: Este artigo é publicado na modalidade Acesso Aberto sob a licença Creative Commons Atribuição 4.0 (CC-BY 4.0), a qual permite uso irrestrito, distribuição, reprodução em qualquer meio, desde que o autor e a fonte sejam devidamente creditados. 